\title{
Extraskeletal osteosarcoma of the lung following treatment of primary small-cell lung carcinoma with chemoradiotherapy: A case report
}

\author{
KEI TAKAMURA ${ }^{1}$, TAKAHIRO OGI ${ }^{1}$, MAKOTO YAMAMOTO ${ }^{1}$ and KEISUKE KIKUCHI ${ }^{2}$ \\ ${ }^{1}$ First Department of Medicine and ${ }^{2}$ Department of Pathology, \\ Obihiro Kosei General Hospital, Obihiro, Hokkaido 080-0016, Japan
}

Received October 5, 2017; Accepted November 15, 2017

DOI: $10.3892 /$ mco.2017.1500

\begin{abstract}
Primary pulmonary osteosarcoma is a rare entity, with only a few cases reported in the literature to date. Moreover, secondary extraskeletal osteosarcoma of the lung following chemoradiotherapy is extremely rare and, to the best of our knowledge, this is the first reported case. We herein present the case of an 80-year-old male patient with small-cell lung cancer (SCLC), who developed what it was initially considered as recurrence of the tumor after chemoradiotherapy. The patient eventually succumbed to the disease, and on autopsy it was discovered that the lung tumor was not in fact SCLC, but rather a secondary osteosarcoma. Osteosarcoma metastasis to the lung is very rare, but must be considered in the differential diagnosis when there is relapse following treatment for primary SCLC.
\end{abstract}

\section{Introduction}

We encountered a case of extraskeletal osteosarcoma of the lung following chemoradiotherapy for small-cell lung carcinoma (SCLC). Primary pulmonary osteosarcoma is a rare entity, with only a few cases reported in the literature to date (1-5). Miller et al (6) reported the incidence of primary pulmonary osteosarcoma as $0.01 \%$. Niimi et al (7) reviewed the 20 cases of primary pulmonary osteosarcoma, and the age on onset was 33-81 years old, male/female ratio was 1:1 and the most common site was left lung. Most of patient died within a year on onset symptom. Moreover, secondary osteoskeletal osteosarcoma of the lung following chemoradiotherapy is extremely rare and, to the best of our knowledge, this is the first case reported to date.

Correspondence to: Dr Kei Takamura, First Department of Medicine, Obihiro Kosei General Hospital, West 6 East 8, Obihiro, Hokkaido 080-0016, Japan

E-mail:keitak@hokkaido.med.or.jp

Key words: osteosarcoma, lung, autopsy

\section{Case report}

An 80-year-old male patient had been diagnosed with SCLC of middle lower lobe of the right lung (Fig. 1A) via brushing cytology at the Obihiro Kosei General Hospital (Obihiro, Japan) in December 2001 and treated with chemoradiotherapy (cisplatin, $80 \mathrm{mg} / \mathrm{m}^{2}, 130 \mathrm{mg}$, Day 1; etoposide, $100 \mathrm{mg} / \mathrm{m}^{2}$, $170 \mathrm{mg}$, Day 1-3) and accelerated hyperfractionation (AHF) at a dose of $45 \mathrm{~Gy} / 30 \mathrm{fr}$. After 4 cycles of chemotherapy, a partial response to the treatment was observed (Fig. 1B). However, the disease recurred locally 3 years later, and the patient was again admitted to our hospital for second-line chemotherapy. The patient's chest computed tomography (CT) scan revealed an oval-shaped tumor in the same region of the lung that had been previously affected (Fig. 1C). The lesion was not evaluated by pathological examination, as its location was the same as that at the initial diagnosis of SCLC, thus, it was considered to be a recurrence of the primary tumor and it was treated with carboplatin (AUC $=4,330 \mathrm{mg}$, Day 1) and irinotecan (CPT-11; $60 \mathrm{mg} / \mathrm{m}^{2}, 100 \mathrm{mg}$, Day 1, 8 and 15) as second-line chemotherapy. As the cancer did not respond sufficiently to this treatment, third-line amrubicin hydrochloride (AMR; $30 \mathrm{mg} / \mathrm{m}^{2}, 50 \mathrm{mg}$, Day 1-3) was administered. Unfortunately, the patient's performance status (PS) decreased, despite treatment. Chemotherapy was discontinued after the first cycle and followed by best supportive care (BSC). During the second hospitalization for second- and third-line chemotherapy, the patient developed a cerebral infarction, causing permanent paralysis in the lower half of his body.

The patient was again admitted to our hospital after sustaining a second cerebral infarction. At that point, his PS was 4 , and retention of the pleural effusion in the right thoracic cavity was detected. A chest tube was inserted to drain the fluid from the lungs; however, the cytology of the pleural effusion was not examined and the patient was clinically diagnosed with pleuritis carcinomatosa. Treatment for a pleural adhesion using OK-432 was administered, which exacerbated his general condition. The patient ultimately succumbed to cancer progression in January 2005. The patient diagnosed as SCLC in December 2001, treated with chemoradiotherapy for 4 months. Local recurrence was observed in January 2004, second and third line chemotherapy was enforced in February 
A

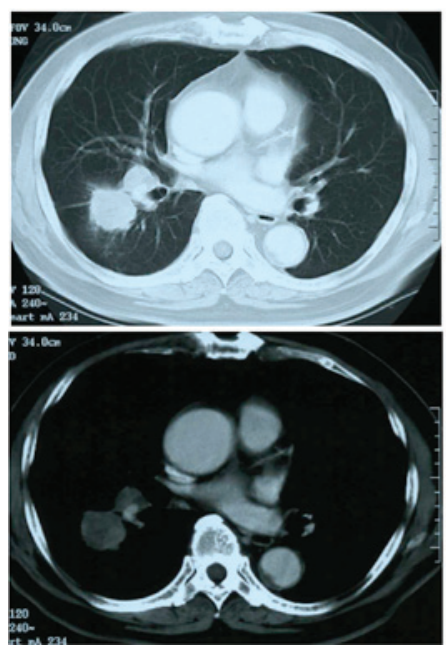

B

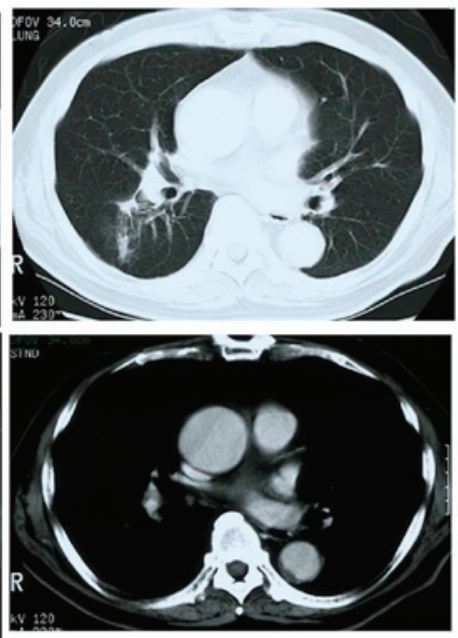

$\mathrm{C}$

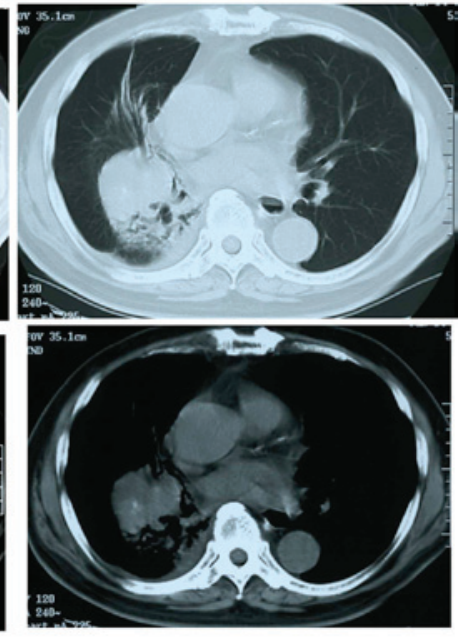

Figure 1. (A) Chest computed tomography scan at initial diagnosis, (B) after chemo-radiotherapy and (C) after recurrence (top, mediastinal window; bottom, lung window).

to April 2004 but the effect was no change. The patient's PS decreased and had a first cerebral infarction in May 2004. BSC then followed, but second cerebral infarction occurred in June 2004. The patient was also diagnosed clinically as pleuritis carcinomatosa, and pleural adhesion was treated with OK-432. The patient died of cancer progression in January 2005.

A postmortem examination was performed, and a large tumor measuring $14 \mathrm{~cm}$ was identified in the middle lower lobe of the right lung (Fig. 2A). On the cut surface, the tumor was grayish-white and focally necrotic. Histologically, the lung parenchyma was diffusely infiltrated by atypical spindle to round cells, with lacy osteoid matrix, bone trabeculae, focal chondroid differentiation, and osteoclastic giant cells (Fig. 2B). Although the right pleura, diaphragm, right bronchus and primary hilum were macroscopically affected by the tumor, the ribs and vertebral bones appeared to be intact. Extrathoracic metastasis was not detected. Immunohistochemically, the tumor cells were positive for vimentin, but negative for pan-cytokeratin (Fig. 2C). These findings were compatible with osteoblastic-type extraskeletal osteosarcoma. Despite thorough histological examination, SCLC was not detected in the autopsy material.

The initial brushing cytology revealed clusters of undifferentiated small cells with a high nuclear-cytoplasmic ratio and dense hyperchromatic nuclei, which are findings typical of SCLC (Fig. 3A). No sarcomatous components were found on retrospective examination; additionally, these cells were positive for pan-cytokeratin (Fig. 3B), but immunohistochemically negative for vimentin, which is the opposite of what was observed in the autopsy material.

\section{Discussion}

In the case presented herein, the patient was diagnosed with SCLC and was treated with chemoradiotherapy. Clinically, the patient responded partially to the treatment. However, the tumor relapsed and additional chemotherapy was administered. The tumor did not respond to second-line chemotherapy, and the PS of the patient deteriorated. BSC was selected as the next mode of treatment; however, the patient succumbed to disease progression. A CT scan revealed that the new lesion was in the same location as the primary tumor, and it was initially diagnosed as SCLC recurrence. However, SCLC tissue was not identified on postmortem examination, and the tumor was instead diagnosed as osteosarcoma. Therefore, this is an extremely rare case of extraskeletal osteosarcoma of the lung following treatment for SCLC with chemoradiotherapy.

The main question is why osteosarcoma occurred in the thoracic cavity following chemoradiotherapy for the primary lung carcinoma. To explain this, three possibilities were considered as follows: The first possibility is that the tumor was a mixed carcinoma and sarcoma. Primary pulmonary osteosarcomas are generally extremely rare, with only a few cases reported in the literature to date (1-5). The origin of the carcinoma in most cases of primary pulmonary carcinosarcoma is non-SCLC (8). Cases involving the combination of SCLC and sarcoma have been reported, but the primary sites for these tumors were the urinary tract or gallbladder $(9,10)$, rather than the lungs. In the present case, the first diagnosis was based on the cytology of bronchoscopically obtained material, so it is possible that the tumor sample was insufficient to detect the sarcomatous components. However, the cytology findings revealed proliferation of tumor cells with small nuclei and epithelial intercellular connections, whereas no sarcomatous component was detected. Immunohistochemical examination was positive for cytokeratin, a marker of epithelial cells. The patient's tumor was reduced in size following chemoradiotherapy for SCLC. In addition, CPT-11 and AMR were administered as secondand third-line chemotherapy, respectively, which were unable to suppress tumor progression. This patient initially responded to anticancer therapy for SCLC, suggesting that he had primary SCLC or carcinosarcoma of the lung, in which the SCLC component was dominant at the initial diagnosis.

The second possibility is histological transformation of the carcinoma to sarcoma. Another case in which SCLC transformed into another type of carcinoma after chemotherapy has 
A

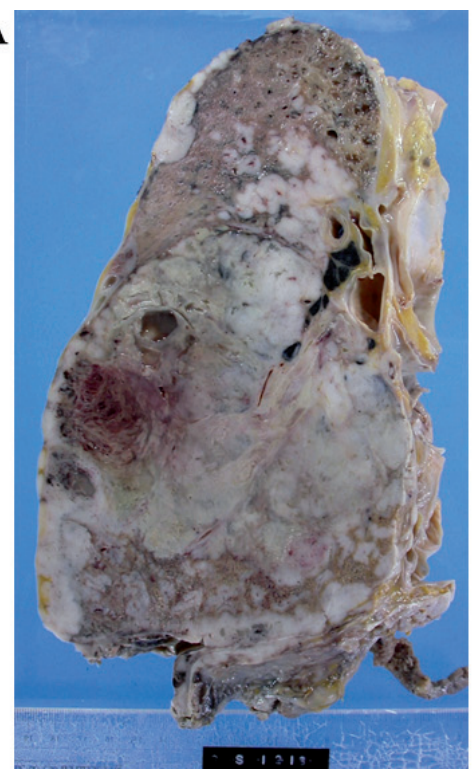

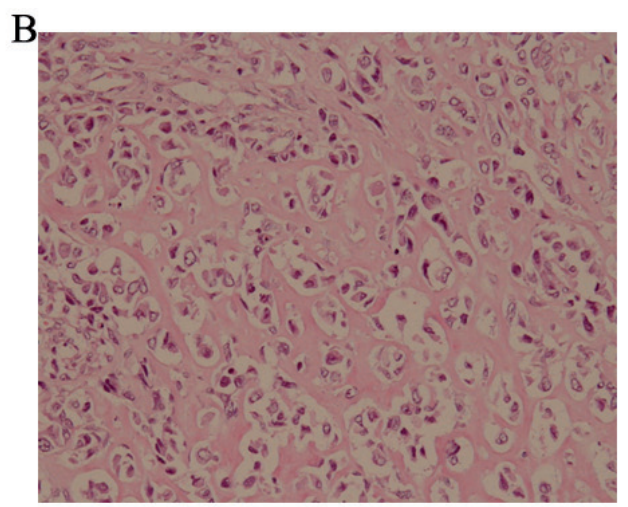

H\&E, x200

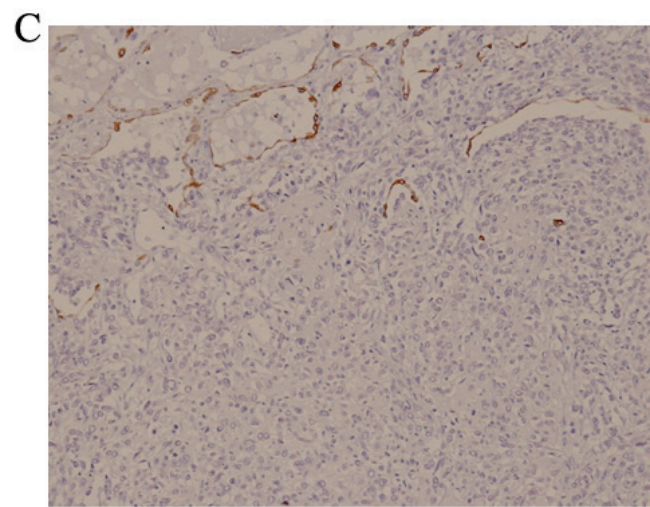

Cytokeratin

Figure 2. (A) Macroscopic specimen. (B) The tumor included lacy osteoid matrix, bone trabeculae, focal chondroid differentiation and osteoclastic giant cells. [Hematoxylin and eosin staining (H\&E); magnification, x200]. (C) On immunohistochemical examination, the tumor cells were negative for pan-cytokeratin (magnification, $\mathrm{x} 200$ ).
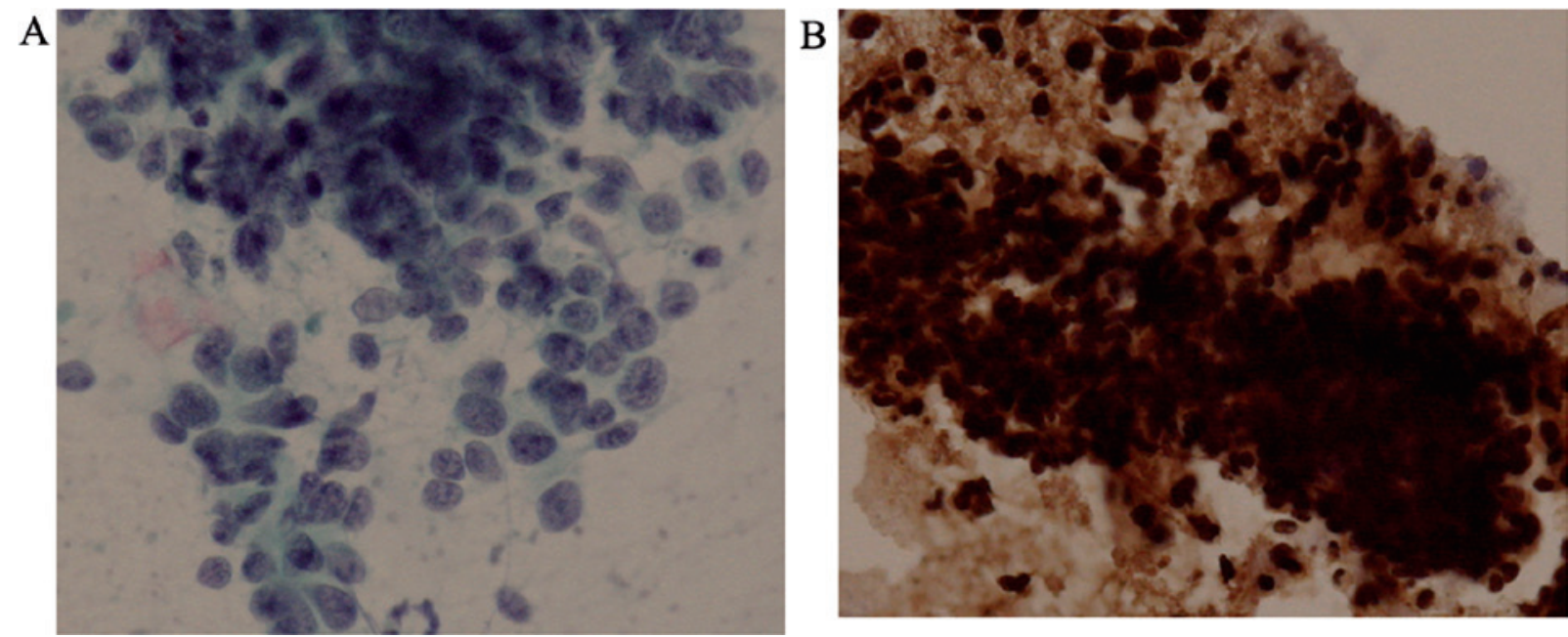

Figure 3. (A) Initial brushing cytology showing clusters of undifferentiated small cells with a high nuclear/cytoplasmic ratio and dense hyperchromatic nuclei, characteristic of small-cell car-cinoma. (B) The cells were positive for pan-cytokeratin.

been reported (11). In a number of cases, however, transformation to other histological types, such as adenocarcinoma, squamous cell carcinoma, and large-cell carcinoma has been reported. Transformation from an epithelial tumor, such as SCLC, to a mesenchymal tumor is extremely rare and, to the best of our knowledge, such a case has not been reported to date. There is a possibility that the chemoradiotherapy promoted a gene mutation, with subsequent transformation of carcinoma to sarcoma.

The last possibility involves the effects of radiotherapy. Cahn et al studied post-irradiation osteosarcoma occurring within the irradiation area after a latency period of several years. The histology of the tumors examined later in this period was found to be different from those of the primary lesion (12). In the present case, the post-irradiation osteosarcoma fulfilled two of these criteria. However, in the majority of the cases, osteosarcoma occurs in bone and soft tissue, rather than in the vital organs. Kondo et al reported a case of pleuropulmonary blastoma with accelerated cartilaginous differentiation following chemotherapy and radiation (13). In that study, the authors suggested the possibility that this treatment was the cause of the cartilaginous differentiation.

It is hypothesized that the tumor described herein was a mixed carcinoma and sarcoma, or was due to a gene mutation promoting the transformation of SCLC to osteosarcoma precipitated by chemoradiotherapy. In conclusion, it is necessary to take into consideration the possibility of osteosarcoma when recurrence of primary lung carcinoma occurs following chemoradiotherapy treatment. Further examination of this pathological entity is required to fully elucidate the mechanism underlying the occurrence of this phenomenon. 
Written informed consent was obtained from the patient for publication of this case report and accompanying images, and no conflict of interest was declared.

\section{References}

1. Chapman AD, Pritchard SC, Yap WW, Rooney PH, Cockburn JS, Hutcheon AW, Nicolson MC, Kerr KM and McLeod HL: Primary pulmonary osteosarcoma: Case report and molecular analysis. Cancer 91: 779-784, 2001.

2. Tatsuaki W, Takashi I, Jotaro S, et al: Primary osteosarcoma of the lung. Nihon Kokyuki Geka Gakkai Zasshi Jpn 49: 823-827, 2010 (In Japanese).

3. Kadowaki T, Hamada H, Yokoyama A, Katayama H, Abe M, Nishimura K, Tomiyama N, Kito K, Miyazaki T and Higaki J: Two cases of primary pulmonary osteosarcoma. Intern Med 44: 632-637, 2005 .

4. Nakata Y, Kubota A, Ueno Y, et al: An autopsy case of Primary pulmonary ostegenic sarcoma. Byori Rinsho Jpn 5: 87-90, 1987 (In Japanese).

5. Niimi R, Matsumine A, Kusuzaki K, et al: Primary osteosarcoma of the lung: A case report. Seikeigeka Jpn 55: 313-316, 2005 (In Japanese).

6. Miller DL and Allen MS: Rare pulmonary neoplasms. Mayo Clin Proc 68: 492-498, 1993.

7. Niimi R, Matsumine A, Kusuzaki K, Inada Y, Kato Y, Maeda M and Uchida A: Primary osteosarcoma of the lung: A case report and review of the literature. Med Oncol 25: 251-255, 2008.
8. Koss MN, Hochholzer L and Frommelt RA: Carcinosarcomas of the lung: A clinicopathologic study of 66 patients. Am J Surg Pathol 23: 1514-1526, 1999.

9. Takahashi Y, Fukushima J, Fukusato T and Shiga J: Sarcomatoid carcinoma with components of small cell carcinoma and undifferentiated carcinoma of the gallbladder. Pathol Int 54: 866-871, 2004.

10. Ishida M, Iwai M, Yoshida K, Kagotani A and Okabe H: Sarcomatoid carcinoma with small cell carcinoma component of the urinary bladder: A case report with review of the literature. Int J Clin Exp Pathol 6: 1671-1676, 2013.

11. Le Vu B, de Vathaire F, Shamsaldin A, Hawkins MM, Grimaud E, Hardiman C, Diallo I, Vassal G, Bessa E, Campbell S, et al: Radiation dose, chemotherapy and risk of osteosarcoma after solid tumours during childhood. Int J Cancer 77: 370-377, 1998.

12. Cahan WG, Woodard HQ, Higinbotham NL, Stewart FW and Coley BL: Sarcoma arising in irradiated bone: Report of eleven cases 1948. Cancer 82: 8-34, 1998.

13. Kondo Y, Anami Y, Iijima T, Noguchi M, Watanabe M and Kaneko M: A case of pleuropulmonary blastoma with accelerated cartilage differentiation after chemotherapy and radiational therapy. Jap J Lung Cancer 46: 167-169, 2006 (In Japanese). 